\title{
Avaliação dos principais fármacos utilizados para o sars-cov-2: uma prospecção cientifica e tecnológica
}

Evaluation of the main pharmacies used for the sars-cov-2: a scientific and technological prospection

Evaluación de los principales farmacoses utilizados para sars-cov-2: una prospección científica y tecnológica

Kelly Maria Rêgo da Silva ORCID: https://orcid.org/0000-0003-3070-4060 Laboratório Central de Saúde Pública do Piauí, Brasil E-mail: kelly-rego@bol.com.br

Pryscilla Bizerra da Silva ORCID: https://orcid.org/0000-0002-5766-2000 Universidade Mauricio de Nassau, Brasil E-mail: pryscillasilva@credishop.com.br

Higo José Neri da Silva

ORCID: https://orcid.org/0000-0003-2089-4147

Faculdade de Tecnologia de Teresina, Brasil

E-mail: higoneri@gmail.com

Pauliana Texeira da Silva

ORCID: https://orcid.org/0000-0003-0358-8025 Faculdade SEVEN, Brasil

E-mail: paulianateixeiras2513@gmail.com

Bruna Layra Silva

ORCID: https://orcid.org/0000-0002-7385-0095 Universidade Estadual do Piauí, Brasil

E-mail: biobiomed.brunalayra@gmail.com

Ana Maria Castro Ferreira

ORCID: https://orcid.org/0000-0003-4378-2893

Universidade de Campinas, Brasil

E-mail: castroferreiraana@gmail.com

Wallyson André dos Santos Bezerra

ORCID: https://orcid.org/0000-0002-9151-9654

Universidade Federal do Maranhão, Brasil

E-mail:w_andre168@hotmail.com

Denilson de Araújo e Silva

ORCID: https://orcid.org/0000-0001-5401-3462 Centro Universitário Uninovafapi, Brasil E-mail: bmdenilsonaraujo@outlook.com

Mariel Rodrigues de Campos

ORCID: https://orcid.org/0000-0002-7253-2212

Universidade Mauricio de Nassau, Brasil E-mail: maryel_r@hotmail.com

Deylane Menezes Teles e Oliveira

ORCID: https://orcid.org/0000-0002-5516-9167

Universidade Federal do Piauí, Brasil

E-mail: deylane.teles@gmail.com

Antonia Luzia Lima do Nascimento

ORCID: https://orcid.org/0000-0001-9740-5669

Universidade Federal do Rio Grande do Sul, Brasil

E-mail: antonialuzialimaa@gmail.com

Alice Maria de Castro Ribeiro

ORCID: https://orcid.org/0000-0002-3506-8772

Instituto Federal do Piaú, Brasil

E-mail: alyce-mcr@hotmail.com

Amanda Miranda da Silva

ORCID: https://orcid.org/0000-0001-6476-4809

Laboratório de Pesquisas em Leishmaniose, Brasil E-mail:miraanda.sa@gmail.com 
Emille Andrade Sousa

ORCID: https://orcid.org/0000-0003-4311-1010 Laboratório de Pesquisas em Leishmaniose, Brasi E-mail: emilleasousa@gmail.com

Iara Katrynne Fonsêca Oliveira ORCID: https://orcid.org/0000-0003-2775-5385 Universidade Federal do Piaú, Brasil E-mail: iarakatrynne@hotmail.com

Michelle Maria Ferreira Lopes ORCID: https://orcid.org/0000-0002-2745-5828 Laboratório de Pesquisas em Leishmaniose, Brasil E-mail: michellef113@hotmail.com

Nathália Batista de Andrade

ORCID: https://orcid.org/0000-0002-9338-6613 Laboratório de Pesquisas em Leishmaniose, Brasil E-mail: veterinarianba@gmail.com

Iluska Martins Pinheiro

ORCID: https://orcid.org/0000-0002-8160-9787 Universidade Mauricio de Nassau, Brasil E-mail: iluskasmartins@gmail.com

Kivia Ludmilla Carvalho Ferreira ORCID: https://orcid.org/0000-0003-4010-9927 DSEI Kaiapó do Pará, Brasil E-mail: kivialudmilla @hotmail.com

Denis Romulo Leite Furtado

ORCID: https://orcid.org/0000-0002-6266-1061 Universidade Estadual do Maranhão, Brasil E-mail: denisfarmac@gmail.com

\begin{abstract}
Resumo
Os Coronavírus são vírus que causam infecções respiratórias podendo afetar animais e humanos. A epidemia de SARSCoV-2, causador da COVID-19 teve início em Wuhan (China) em dezembro de 2019, e se espalhou por diversos países tornando-se uma pandemia perigosa e sem tratamento especifico. Esse trabalho pretende mostrar casos tratados com os principais fármacos para o SARS-CoV-2 avaliando sua eficácia e desvantagens. Foram utilizadas publicações com dados de tratamentos farmacológicos para o novo SARS-CoV-2, publicações cientificas, oficiais da Organização Mundial da Saúde (World Health Organization - WHO) e governamentais, dentre a data (2019-2020). Os dados foram coletados nas bases cientificas: LILACS, PUBMED, PERIODICOS, GOOGLE ACADEMICOS, SCIENCE DIRECT, BIRAME, Biblioteca Virtual em saúde - BVS, SCIELO, Cochrane Library, HighWire Press, Scopus e Elsevier; e as bases tecnológicas: WIPO e INPI. A utilização desses fármacos pode ter ocasionado melhora e cura de pacientes globalmente. Acredita-se que são eficazes contra a doença. Porém, temos a necessidade de comprovações cientificas. Nos resultados observamos os fármacos utilizados mundialmente e as patentes aprovadas até (15/09/2020). Não existe tratamento para a Covid19, utilizou-se medicamentos amenizadores dos sintomas gripais da doença. Nos casos emergenciais foram utilizados a combinação do antibiótico azitromicina e hidroxicloroquina, usados para malária e outros medicamentos. Este trabalho avaliou grande parte dos medicamentos que estão em uso para o tratamento do SARS-CoV-2, com o intuito de apresentar um fármaco ou mais que seja eficaz para o tratamento da doença, mostrando ainda se foram realizadas pesquisas in vitro e in vivo na busca de uma melhor tratativa.
\end{abstract}

Palavras-chave: Coronavírus; Covid-19; SARS-CoV-2.

\begin{abstract}
Coronaviruses are viruses that cause respiratory infections that can affect animals and humans. The SARS-CoV-2 epidemic, which caused COVID-19, began in Wuhan (China) in December 2019, and spread to several countries, becoming a dangerous pandemic without specific treatment. This work aims to show cases treated with the main drugs for SARS-CoV-2, evaluating its efficacy and disadvantages. Publications with data from pharmacological treatments for the new SARS-CoV-2, scientific publications, world health organization (WHO) and governmental publications were used, among the date (2019-2020). Data were collected from the following scientific databases: LILACS, PUBMED, PERIODICOS, GOOGLE ACADEMICOS, SCIENCE DIRECT, BIRAME, Virtual Health Library - VHL, SCIELO, Cochrane Library, HighWire Press, Scopus and Elsevier; and the technological bases: WIPO and PTO. The use of these drugs may have led to improvement and cure of patients globally. They are believed to be effective against the disease. However, we need scientific proofs. In the results we observed the drugs used worldwide and the patents approved until (15/09/2020). There is no treatment for Covid19, medications that soften the flu-like symptoms of the disease were used. In emergency cases, the combination of the antibiotic azithromycin and hydroxychloroquine, used for malaria and other medicines, was used. This study evaluated most of the drugs that are in use for the treatment of SARS-CoV-2, in order to present a drug or more that is effective for the treatment of the disease, also showing whether in vitro and in vivo research was conducted in the search for a better treatment.
\end{abstract}


Keywords: Coronavirus; Covid-19; SARS-CoV-2.

\section{Resumen}

Coronaviruses are viruses that cause respiratory infections that can affect animals and humans. The SARS-CoV-2 epidemic, which caused COVID-19, began in Wuhan (China) in December 2019, and spread to several countries, becoming a dangerous pandemic without specific treatment. This work aims to show cases treated with the main drugs for SARS-CoV-2 evaluating its efficacy and disadvantages. Publications with data from pharmacological treatments for the new SARS-CoV-2, scientific publications, world health organization (WHO) and governmental publications were used, among the date (2019-2020). Data were collected from the following scientific databases: LILACS, PUBMED, PERIODICOS, GOOGLE ACADEMICOS, SCIENCE DIRECT, BIRAME, Virtual Health Library - VHL, SCIELO, Cochrane Library, HighWire Press, Scopus and Elsevier; and the technological bases: WIPO and PTO. The use of these drugs may have led to improvement and cure of patients globally. They are believed to be effective against the disease. However, we need scientific proofs. In the results we observed the drugs used worldwide and the patents approved until (15/09/2020). Los coronavirus son virus que causan infecciones respiratorias que pueden afectar a animales y seres humanos. La epidemia del SARS-CoV-2, que causó covid-19, comenzó en Wuhan (China) en diciembre de 2019, y se extendió a varios países, convirtiéndose en una peligrosa pandemia sin tratamiento específico. Este trabajo tiene como objetivo mostrar los casos tratados con los principales fármacos para el SARS-CoV-2 evaluando su eficacia y desventajas. Entre la fecha (2019-2020) se utilizaron publicaciones con datos de tratamientos farmacológicos para el nuevo SARS-CoV-2, publicaciones científicas, organización mundial de la salud (OMS) y publicaciones gubernamentales.

Palabras clave: Coronavirus; Covid-19; SARS-CoV-2.

\section{Introdução}

O coronavírus foi descoberto por volta do século XXI. O novo SARS-CoV da síndrome respiratória aguda possuiu uma letalidade de 10\% em 2003 e chegou em cinco continentes. Já o MERS-CoV em 2012, possuiu uma mortalidade de 35\% na península Arábica. Acreditava-se que o vírus fosse apenas zoonótico, sendo seus principais hospedeiros o morcego, dromedário e civeta (Wu et al. 2020).

O vírus citado é da família coronaviridae; e tem como principal sintomas a Covid-19 que causa febre, tosse e dificuldade em respirar. Sua transmissão ocorre geralmente através de tosse ou espirro, contato pessoal próximo (toque ou aperto de mão), contato com objetos ou superfícies contaminadas, seguido então de contato com a boca, nariz ou olhos (Brasil, 2020).

Os Coronavírus são vírus envelopados que contém o material genético (RNA) no seu interior. São da família Coronaviridae possuem três gêneros $\alpha, \beta, \gamma$ e $\delta$. O COVID19 recentemente modificado para SARS-CoV-2, faz parte do gênero $\beta$, ele possui proteínas estruturais (S, E, M, N) (Wu et al. 2020).

A mutação desse vírus se tornou uma pandemia mundial, gerando o caos em diversos continentes, provocando crise econômica e de saúde pública principalmente. O COVID19 possui uma mortalidade de aproximadamente 2\% no mundo (até início de abril de 2020). Ainda é uma doença pouco conhecida no mundo, era apenas uma zoonose até possuir uma mutação e atingir humanos em dezembro de 2019 no continente Asiático, se disseminando em vários continentes. Atualmente o principal método diagnóstico é o método de reação em cadeia da polimerase com transcrição reversa (RT-PCR), método muito eficaz e rápido (Filho et al. 2020).

As Infecções Respiratórias Agudas (IRA'S) estão diretamente relacionadas ao contágio por vírus e são responsáveis por grande parte da mortalidade e morbilidade humana, sendo um dos sintomas diretamente ligado à infecção de Covid 19 causada pelo coronavírus. É observado que a maioria destes indivíduos possuem doenças crônicas associadas, como: diabetes, obesidade, cardiopatia, dentre outros (Nobre et al 2014).

$\mathrm{Na}$ época da segunda Guerra Mundial já havia surgido a necessidade de elaborar um medicamento eficaz que fosse utilizado para tratar um vírus perigoso, a malária, onde originou-se a cloroquina primaquina, pirimetamina e a partir da paludrina, medicamentos que representam um grande marco científico. Sem muitas opções de tratamento para o coravírus na atualidade, realiza-se testes com diversos medicamentos como antivirais e antibióticos. Uma das combinações medicamentosas que está 
sendo utilizada é a combinação de hidroxicloroquina (medicamento utilizado para a malária, lúpus e outras doenças) associada a Azitromicina (antibiótico). Mediante a isso precisamos demonstrar as vantagens desses fármacos para o SARS-CoV-2 (Filho et al. 2020).

Até o presente momento não existe tratamento ou vacina específico para tratar ou curar os indivíduos infectados com o vírus. Porém, alguns medicamentos estão sendo utilizados com cautela e os seus efeitos tem sido observados minunciosamente, na busca de comprovações cientificas dos fármacos em uso. Muitos testes estão sendo feitos também in vitro com medicamentos que já existem para tratamento de outras patologias. As principais terapias para o coronavirus são divididas em duas categorias dependendo do seu alvo, atuando ou no sistema imunológico ou diretamente nas células. O MERS liga-se ao receptor DPP4 já o SARS ao receptor da enzima de conversão da angiotensina 2. Por não ter tratamento especifico, estão sendo testados inicialmente antivirais de amplo espectro já existentes. Cada vez mais tem se intensificado os testes feitos com a hidroxicloroquina e a azitromicina (Wu et al. 2020).

Este trabalho avaliou grande parte dos medicamentos que estão em uso para o tratamento do SARS-CoV-2, com o intuito de apresentar um fármaco ou mais que seja eficaz para o tratamento da doença, mostrando ainda se foram realizadas pesquisas in vitro e in vivo na busca de uma melhor tratativa.

\section{Metodologia}

Trata-se de uma pesquisa de revisão integrativa, com objetivo analítico e explicativo com abordagem qualitativa. Essa pesquisa busca através de publicações cientificas descrever seus resultados, explicando suas causas e efeitos. Sua abordagem implica que tudo o que for realizado será qualificado para melhor demonstração dos resultados obtidos pela pesquisa. Onde foi utilizado programa Prima para melhor distribuição e interpretação dos dados estatísticos (Pereira et al., 2018).

Foram utilizadas todas as publicações que possuírem dados de tratamentos farmacológicos para o novo SARS-CoV-2, publicações cientificas, oficiais da Organização Mundial da Saúde (World Health Organization - WHO) e governamentais, dentre a determinada data (2019-2020).

Os dados foram coletados utilizando as bases cientificas: Literatura Latino-Americana e do Caribe em Ciências da Saúde (LILACS), National Library of medicine (PUBMED), PERIODICOS, SCIENCE DIRECT, Biblioteca Virtual em saúde - (BVS), Scientific Eletronic Library Online (SCIELO), Cochrane Library, HighWire Press, Scopus e Elsevier; e as bases tecnológicas: World Intellectual Property Organization (WIPO) e Instituto Nacional de Propriedade Industrial (INPI).

Foram analisados os trabalhos publicados e comparados para maior relevância dos resultados. Avaliando os tratamentos utilizados pelo mundo.

Foram utilizados nessa pesquisa todos os trabalhos que relatam tratamento e cura do novo coronavírus, publicados nos anos de 2019 e 2020, nos idiomas: inglês, espanhol e português. Foram excluídos todos os demais trabalhos e publicações que não se referem ao estudo do novo coronavirus (Covid - 19).

A pesquisa não apresentou nenhum risco, pois todos os dados coletados são ofertados pelas bases cientificas, onde não existiu contato nenhum com os indivíduos infectados e não se fizeram divulgações dos dados pessoais dos indivíduos envolvidos em tais estudos. A pesquisa é voltada a análise de dados para avaliação da eficácia dos medicamentos utilizados até o presente momento para tratamento ou cura do coronavírus.

\section{Resultados e Discussão}

Foram encontrados um total de 21 artigos (tabela 1) nas bases cientificas: Literatura Latino-Americana e do Caribe em Ciências da Saúde (LILACS), National Library of medicine (PUBMED), PERIODICOS, SCIENCE DIRECT, Biblioteca Virtual 
em saúde - (BVS), Scientific Eletronic Library Online (SCIELO), Cochrane Library, HighWire Press, Scopus e Elsevier; e 1 na bases tecnológica: World Intellectual Property Organization (WIPO) não tivemos patentes para a base do Instituto Nacional de Propriedade Industrial (INPI). Foram utilizados como descritores: CORONAVIRUS, SARS-CoV-2 E COVID 19, nos idiomas inglês, português e espanhol. Onde obtivemos os artigos abaixo descritos na tabela 1 e discutidos em forma de tópicos relacionados com o tema.

Tabela 1. Artigos encontrados e selecionados.

\begin{tabular}{|c|c|c|c|}
\hline & AUTORES & ARTIGOS & $\overline{\text { ANO }}$ \\
\hline 1 & AMARAL, J.L.G. et al. & $\begin{array}{l}\text { Intervenção com cloroquina/hidroxicloroquina com } \\
\text { ou sem azitromicina para COVID-19 (SARS-Cov 2): } \\
\text { sinopse baseada em evidências. }\end{array}$ & 2020. \\
\hline 2 & WU, C; LIU, et al & $\begin{array}{l}\text { Analysis of therapeutic targets for SARS-CoV-2 and } \\
\text { discovery of potential drugs by computational } \\
\text { methods. }\end{array}$ & 2020. \\
\hline 3 & $\begin{array}{l}\text { SECRETARIA DE } \\
\text { ATENÇÃO PRIMÁRIA À } \\
\text { SAÚDE (SAPS) }\end{array}$ & $\begin{array}{l}\text { Ministério da Saúde. Protocolo de manejo clínico do } \\
\text { coronavírus (COVID-19) na atenção primária à } \\
\text { saúde. }\end{array}$ & 2020 \\
\hline 4 & SANDLER, R. & $\begin{array}{l}\text { FDA aprova cloroquina e hidroxicloroquina para } \\
\text { tratamento emergencial de coronavírus. }\end{array}$ & 2020. \\
\hline 5 & $\begin{array}{l}\text { DELLA-TORRE, E.; } \\
\text { CAMPOCHIARO, C; } \\
\text { CAVALLI, G. et al }\end{array}$ & $\begin{array}{l}\text { Interleukin-6 blockade with sarilumab in severe } \\
\text { COVID-19 pneumonia with systemic } \\
\text { hyperinflammation: an open-label cohort study. }\end{array}$ & 2020. \\
\hline 6 & NOBRE, A. F. S. et al. & $\begin{array}{l}\text { Primeira detecção de coronavírus humano associado } \\
\text { à infecção respiratória aguda na Região Norte do } \\
\text { Brasil. }\end{array}$ & 2014 \\
\hline 7 & $\begin{array}{l}\text { SATHYAMOORTHY, } \\
\text { N.K.; CHINTAMANENI, } \\
\text { P.K.; CHINNI, S. }\end{array}$ & $\begin{array}{l}\text { Plausible role of combination of Chlorpromazine } \\
\text { hydrochloride and Teicoplanin against COVID- } 19 .\end{array}$ & 2020 \\
\hline 8 & $\begin{array}{l}\text { SECRETARIA DO } \\
\text { ESTADO DE SÃO } \\
\text { PAULO. }\end{array}$ & $\begin{array}{l}\text { Protocolo laboratorial para a coleta, } \\
\text { acondicionamento e transporte de amostras biológicas } \\
\text { para investigação de COVID-19. }\end{array}$ & 2020 \\
\hline 9 & $\begin{array}{l}\text { STEBBING, J.; PHELAN, } \\
\text { A; GRIFFIN, I. et al. }\end{array}$ & $\begin{array}{l}\text { COVID-19: combining antiviral and anti- } \\
\text { inflammatory treatments. }\end{array}$ & 2020. \\
\hline 10 & $\begin{array}{l}\text { GEORGE, P. M. D.R.; } \\
\text { JENKINS, G. }\end{array}$ & $\begin{array}{l}\text { Pulmonary fibrosis and COVID-19: the potential role } \\
\text { for antifibrotic therapy. }\end{array}$ & 2020 \\
\hline 11 & $\begin{array}{l}\text { IMPERADOR, C.H.L.; } \\
\text { ESPREAFICO JUNIOR, }\end{array}$ & $\begin{array}{l}\text { Cloroquina e hidroxicloroquina associado ao zinco } \\
\text { e/ou azitromicina na COVID-19. }\end{array}$ & 2020. \\
\hline
\end{tabular}




\begin{tabular}{|c|c|c|c|}
\hline & $\begin{array}{l}\text { C.R.; NASCIMENTO, } \\
\text { A.M.V. }\end{array}$ & & \\
\hline 12 & $\begin{array}{l}\text { FILHO ARAUJO, J. B.A.; } \\
\text { et al. }\end{array}$ & $\begin{array}{l}\text { Pneumonia por COVID-19: qual o papel da imagem } \\
\text { no diagnóstico? }\end{array}$ & 2020. \\
\hline 13 & LANA; RM, et al & $\begin{array}{l}\text { Emergência do novo coronavírus (SARS-CoV-2) e o } \\
\text { papel de uma vigilância nacional em saúde oportuna } \\
\text { e efetiva. }\end{array}$ & 2020. \\
\hline 14 & MADJD, ZAHRA. & $\begin{array}{l}\text { The Lancet Infectious Diseases Spotlight of } \\
\text { Remdesivir in comparison with Ribavirin, } \\
\text { Favipiravir, Oseltamivir and Umifenovir in } \\
\text { Coronavirus }\end{array}$ & 2019 \\
\hline 15 & $\begin{array}{l}\text { MENEZES, C.R.; } \\
\text { SANCHES, C.; } \\
\text { CHEQUER, F.M.D. }\end{array}$ & $\begin{array}{l}\text { Efetividade e toxicidade da cloroquina e da } \\
\text { hidroxicloroquina associada (ou não) à azitromicina } \\
\text { para tratamento da COVID-19. O que sabemos até o } \\
\text { momento? }\end{array}$ & 2020. \\
\hline 16 & MINISTÉRIO DA SAÚDE & $\begin{array}{l}\text { - Secretária de Vigilância em saúde. Centro de } \\
\text { operações de emergência de saúde pública. Boletim } \\
\text { Epidemiológico Especial - COE }\end{array}$ & 2020. \\
\hline 17 & $\begin{array}{l}\text { SECRETARIA DE } \\
\text { ATENÇÃO PRIMÁRIA À } \\
\text { SAÚDE (SAPS) }\end{array}$ & $\begin{array}{l}\text { Ministério da Saúde. Manejo de corpos no contexto } \\
\text { do novo coronavírus COVID-19. }\end{array}$ & 2020 \\
\hline 18 & $\begin{array}{l}\text { WU, T.C.; SACILOTTO, } \\
\text { L.; DARRIEUX, F.C.C. et } \\
\text { al. }\end{array}$ & $\begin{array}{l}\text { Controle do Intervalo QT para Prevenção de Torsades } \\
\text { de Pointes Durante uso de Hidroxicloroquina e/ou } \\
\text { Azitromicina em Pacientes com COVID } 19 .\end{array}$ & 2020. \\
\hline 19 & CHAN, J.F. et al. & $\begin{array}{l}\text { A familial cluster of pneumonia associated with the } \\
2019 \text { novel coronavirus indicating personto-person } \\
\text { transmission: a study of a family cluster. }\end{array}$ & 2020. \\
\hline 20 & $\begin{array}{l}\text { CENTRO DE } \\
\text { OPERAÇÕES DE } \\
\text { EMERGÊNCIAS EM } \\
\text { SAÚDE PÚBLICA - COE- } \\
\text { COVID }\end{array}$ & $\begin{array}{l}\text { Ministério da Saúde. Plano de Contingência Nacional } \\
\text { para Infecção Humana pelo novo Coronavírus } \\
\text { COVID-19. }\end{array}$ & 2020. \\
\hline 21 & ELFIKY, A & $\begin{array}{l}\text { A.Ribavirin, Remdesivir, Sofosbuvir, Galidesivir, } \\
\text { and Tenofovir against SARS-CoV-2 RNA dependent } \\
\text { RNA polymerase (RdRp): A molecular docking } \\
\text { study. }\end{array}$ & 2020 \\
\hline
\end{tabular}

Fonte: Autores (2020).

\subsection{Histórico da doença}

O novo vírus que causa infecção no trato respiratório pode afetar tanto animais quanto humanos, já foi responsável por outras endemias graves. A epidemia de SARS que ocorreu em 2003, em Hong-Kong, teve letalidade aproximada de 10\% (por 
cento); a Síndrome do Oriente Médio em 2012, ocorreu na Arábia Saudita, onde teve letalidade de 30\% (por cento) atualmente o SARS-CoV-2, responsável da COVID-19 onde teve o primeiro caso no mês de dezembro, na China em Wuhan, onde rapidamente se espalhou por diversos países e continentes se tornando uma pandemia mundial (Lana et al. 2020).

Em Wuhan na China ocorreram os primeiros casos confirmados e os primeiros óbitos, o segundo epicentro ocorreu na Europa, na Itália atingindo também diversos países da Europa como a Espanha, logo após New York Times se tornou o próximo epicentro da doença se tornando uma grande calamidade pública também nas Américas (Ibidem).

Atualmente, a Organização Mundial da Saúde (OMS) registrou 972.640 casos confirmados de COVID-19 com 50.325 óbitos, com quase 5.000 novos óbitos. Os Estados Unidos da América é possui recentemente o maior número de casos confirmados, possuindo um total de 213.600 a Itália, entretanto agrega o maior número de óbitos com 13.917 mortos até a presente data (03 de abril de 2020). No Brasil atualmente possuímos 9.056 casos confirmados, sendo que 1.146 ocorreram durante um período de 24 horas até a presente data (03/04/2020). Onde a região Sudeste (principalmente o estado de São Paulo) apresenta o maior número de casos, sucessivo de Nordeste (com o Ceará), Sul (Distrito Federal), Centro-Oeste (Minas Gerais), e Norte (Amazonas) (Brasil, 2020).

Nos dias atuais o Brasil possui um total de 359 mortes por coronavírus, sendo São Paulo (219), Rio de Janeiro (47), Ceará (22), Pernambuco (10) e Amazonas (7), os estados com maiores óbitos (03/04/2020) (Ibidem).

No estado do Piauí até a presente data (05 de abril de 2020), possui 21 casos confirmados com 4 óbitos, possuindo a maior letalidade do país com 19\% (Ibidem).

O coronavírus era apenas uma zoonose, quando em dezembro de 2019 na China ocorreu a infecção em humanos. A primeira transmissão humana foi ocasionada pelo contato de gotículas oriundas de pacientes sintomáticos, porém existem também os pacientes assintomáticos, esses ainda passando por pesquisas para comprovação de transmissão, o período de transmissão pode variar de 0 a 14 dias (Secretaria de Atenção Primária à Saúde, 2020a).

\subsection{Sintomatologia}

Primeiro ocorre a síndrome gripal, com os sintomas de febre $>37,5$, tosse, dispneia, mialgia e fadiga, sintomas respiratórios superiores, sintomas gastrointestinais, como diarreia (mais raros). Os sintomas podem variar de assintomática a leve, sobretudo para crianças e jovens adultos. Podendo ser mais severa para idosos, pacientes com doenças crônicas e profissionais de saúde, sendo capaz de levar a choque séptico e falência respiratória, na maioria dos pacientes evoluindo par a óbito (Chan et al. 2020).

\subsection{Diagnóstico clínico e laboratorial}

A coleta do material é realizada com a utilização de $3 \mathrm{swab}$ 's, onde será inserido na narina até a nasofaringe realizar movimentos rotatórios para captação de células, metodologia tem que ser repetida nas duas narinas. Já o terceiro swab será inserido na orofaringe, sendo imprescindível não tocar o swab na língua, para não ocorrer contaminação da amostra (Secretaria do Estado De São Paulo, 2020a; Nobre et al. 2014).

O diagnóstico laboratorial ocorre por biologia molecular pelo sequenciamento do RNA viral. Extração do RNA viral (RNAv) ocorre através da amostra clínica, logo após levada para a reação em cadeia mediada pela polimerase precedida de transcrição reversa (RT-PCR) para essa etapa se utiliza grupos controles (positivo e negativo), depois da amplificação é realizado a etapa de Eletroforese em gel de agarose para a visualização dos amplicons. Para a caracterização molecular do CoVh se utiliza a RT-PCR, em seguida a purificação e quantificação do produto da RT-PCR, baseada no método de terminação em cadeia, logo após a Reação de sequenciamento, edição e alinhamento das sequências (Ibidem). 


\subsection{Plano Nacional utilizado para evitar a proliferação do vírus}

A primeira etapa a seguir é o cuidado dos profissionais de saúde para evitarem contato com amostras biológicas de pacientes contaminados, como sangue e fluídos corporais. Se torna extremamente necessário a utilização de EPI’S (Equipamento de proteção individual) (Secretaria do Estado de São Paulo, 2020b).

Não é recomendado realizar tanatopraxia, é necessário embalar o corpo com lençóis, saco impermeável próprio (para tentar impedir vazamento de fluidos corpóreos), já o segundo saco (externo) e desinfetar com álcool a 70\%, solução clorada $0,5 \%$ a $1 \%$ ou outro saneante recomendado pela ANVISA compatível com o material do saco, logo após etiquetado com a identificação do paciente. O corpo deve ser embalado antes do envio para familiares, o serviço funerário deve saber que está levando um corpo com COVID 19 e que se refere a risco 3 (Ibidem).

Em caso de mortes em casa, os familiares não devem de forma nenhuma ter contato com o corpo. Em locais públicos tem que ser acionado imediatamente as autoridades locais (Ibidem).

\subsection{Medicações utilizadas para tratamento}

Ainda não existe uma medicação ou combinação de medicações especificas para tratar a COVID-19. Podem ser utilizados fármacos no intuito de amenizar os sintomas causados pela patologia. Alguns pacientes são tratados com hidroxicloroquina com associação à azitromicina, porém não existe ainda comprovações cientificas sobre a eficácia de suas utilizações, entretanto sua utilização está em observação abrindo caminhos a uma possível eficácia contra o vírus. Sendo assim, esses medicamentos veem estão sendo utilizados em vários países nos casos emergenciais da doença (Sandler, 2020).

Sabe-se que esses fármacos que são citados nesta pesquisa estão sendo utilizados com a intenção de combate ao vírus, mas existe também a utilização de diversos medicamentos para combater apenas os sintomas da doença, esses, não serão mostrados no presente trabalho.

Para complemento, foram analisadas nas bases de patentes: internacional WIPO (World Intellectual Property Organization) (Tabela 2) e na base nacional INPI (Instituto Nacional de Propriedade Industrial). Onde foram encontradas 185 patentes na base internacional, onde apenas uma patente se refere a um possível fármaco, as demais se referem a testes de diagnósticos, criação de equipamento de proteção, e produtos de limpeza para o Covid 2019. Já na base nacional não foram encontrados resultados com as palavras chaves até o momento (15/09/2020).

Tabela 2. Resultado encontrado na base de patente internacional.

\begin{tabular}{ll}
\hline$\underline{111265500}$ & FARMACÊUTICO PARA PREVENIR E TRATAR \\
INT.CLASS A61 K9/72APPL.NO 202010135846. & COVID -19 E SEU MÉTODO DE PREPARAÇÃO \\
& CN - 12.06.2020 \\
\hline 1APPLICANT 广西医科大学第一附属医院INVENTOR 李超乾 \\
E O CUSTO MÉDICO É REDUÇÃO DA COMPOSIÇÃO FARMACÊUTICA PARA PREVENIR E TRATAR \\
COVID -19 É CAPAZ DE PREVENIR E TRATAR EFICAZMENTE COVID -19 A REDUÇÃO DO \\
PACIENTE PARA CRÍTICO OU CRÍTICO DOENTE É MITIGADA. O TEMPO DE TRANSDUÇÃO DE \\
ÁCIDO NUCLEICO FOI REDUZIDO EM CERCA DE 4 DIAS A DURAÇÃO DO TRATAMENTO \\
TAMBÉM É REDUZIDO AO MESMO TEMPO, OUTRAS INFECÇÕES POR INFECÇÃO DO TRATO \\
RESPIRATÓRIO PODEM SER EVITADAS E CONTROLADAS ESPECIFICAMENTE. PARA A \\
PREVENÇÃO CLÍNICA DE COVID -19 A PREVENÇÃO E O TRATAMENTO DE VÍRUS \\
RESPIRATÓRIOS PROPORCIONAM UMA NOVA ABORDAGEM E ESQUEMA \\
\hline
\end{tabular}

Fonte: Autores (2020). 
Na base tecnológica mostra que um possível fármaco está sendo criado especificamente para o tratamento dessa doença, possuindo grande eficácia em pesquisas, gerando assim uma patente. Vários artigos mostram diversos fármacos utilizados para a doença. Foram realizadas as pesquisas de determinadas bases cientificas descritas na metodologia e explanado os resultados de acordo com o fármaco conforme (tabela 3):

Tabela 3. Fármacos utilizados atualmente como possível tratamento para o Covid 19.

\begin{tabular}{l|l}
\hline FÁRMACOS & UTILIZAÇÃO ATUAL \\
\hline Pirfenidona & Utilizado para fibrose pulmonar \\
\hline Ritonavir/ Atazanavir/ Darunavir/ Cobicistat/ & Antirretroviral \\
Realtegravir/ & \\
Tenofovir+emtricitabina/Dolutegravir/ Saquinavir/ & \\
Lamivudina/ Tenofovir/ Lopinavir/ Ritonavir/ & \\
Cobicistrat/ Abacavir/ Etravirine/ Efavirenz/ & \\
Rilpirivina/ Maraviroc/ Bictegravir/ Tipranavir/ & \\
Indinavir/Zidovudina/ Remdesivir & \\
\hline Ribavirina & Hepatite C crônica e outras doenças \\
\hline Cloroquina/Hidroxicloroquina & Malária e outras doenças \\
\hline Sarilumab & Artrite reumatoide \\
\hline Oseltamivir/ Arbidol / Umifenovir & Influenza \\
\hline Azitromicina & \\
\hline Aciclovir & \\
\hline
\end{tabular}

Fonte: Autores (2020).

\subsection{Pirfenidona}

A pirfenidona é um medicamento antifibrótico, utilizado para a tratamento de fibrose pulmonar idiopática considerada de ligeira a moderada. Este fármaco possui diversos modos de ação, é utilizado como terapia para uma maior qualidade de vida dos pulmões, por conta dos seus vários mecanismos de ação, com isso, foi criada a hipótese que ela poderia ser utilizada para o tratamento do Covid 19; sendo assim também por que a Pirfenidona apresenta efeitos que regulam uma série de citocinas, incluindo o fator de crescimento do tecido conjuntivo, eregula também a expressão do receptor da ECA (Enzima Conversora de Angiotensina), que é o principal receptor celular para coronavírus (Wu et al. 2020).

A taxa de complicações de fibrose pulmonar nos hospitais chega a 50\%, que na maioria das vezes são desencadeadas por infecções virais (George, 2020).

Desde o mês de abril desse ano de 2020 a Pirfenidona está disponível na forma oral, sendo assim não são administradas em pacientes intubados ou que utilizam ventiladores mecânicos, tendo desse modo seu uso restringido para pacientes com Covid 19. Pesquisas realizadas em Wuhan mostraram que muitos pacientes que utilizaram esse tratamento apresentaram hepatotoxicidade $(39 \%)$ e lesões renais $(28,8 \%)$ (Ibidem). 


\subsection{Sarilumabe}

Foi administrado o medicamento Sarilumabe em pacientes, onde 61\% desses infermos apresentaram melhora clínica e 7 \% foi a óbito. Esses achados não foram diferentes dos resultados do grupo controle. A taxa de infecção pulmonar foi semelhante aos dois grupos (Seifirad, 2020).

Existem ainda poucas publicações com o tema, é necessário a realização de ensaios clínicos pragmáticos, envolvendo um número maior de pacientes, para que seja possível analisar a efetividade no combate ao coronavírus, bem como a segurança do uso desses fármacos citados (Imperador, 2020).

\subsection{Antirretrovirais}

Os antirretrovirais sendo atualmente estudados para o combate do novo vírus (Covid 19) são eles: Ritonavir/Atazanavir/ Darunavir/ Cobicistat/ Realtegravir/ Tenofovir+emtricitabina/ Dolutegravir/ Saquinavir/ Lamivudina/ Tenofovir/ Lopinavir/ Ritonavir/Cobicistrat/Abacavir/Etravirine/ Efavirenz/ Rilpirivina/ Maraviroc/ Bictegravir/ Tipranavir/Indinavir/Zidovudina/ Remdesivir.

Os medicamentos observados nos quais apresentaram um bom resultado de ligação ao SARS-CoV-2, que contradiz a função da enzima polimerase são: O Galidesivir, Remdesivir, Tenofovir, Sofosbuvir e Ribavirina. Esses medicamentos são utilizados para o tratamento de retrovírus. Porém é necessário ainda, uma análise mais complexa estes fármacos (Elfiky, 2020).

O Setrobuvir teve resultados promissores, exibe uma ligação mais firme que os nucleotídeos ativos. Já a Ribavirina possuí interações únicas com o vírus, o mesmo padrão foi encontrado para o medicamento Tenofovir (Stebbing et al. 2020).

Devido à grande emergência de saúde pública mundial por ser um vírus ainda pouco conhecido, por este motivo não possui um tratamento eficaz até o momento, esses medicamentos estão sendo utilizados por apresentarem um bom resultado in vitro (Della-Torre et al. 2020).

Os alvos dessas drogas são os membros da família quinase associada a entorpecentes, eles demonstraram uma redução da infecção viral. A administração desses medicamentos depende da gravidade do caso clínico do paciente devido a presença do vírus, por serem medicamentos que ainda não foram aprovados para a doença (Elfiky, 2020).

O fármaco Baricitinibe apresnta menos efeitos colaterais e se mostrou mais aceitável devido a sua dosagem de apenas 1 comprimido via oral por dia (Stebbing et al. 2020).

O Remdesivir é um fármaco antiviral de amplo espectro, que também foi utilizado na epidemia provocada pelo vírus Ebola em 2017. Ele tem ação contra familias de RNA incluindo filoviridae, paramyxoviridae, pneumoviridae, vírus Ebola, vírus sincicial respiratório (RSV), vírus hendra e coronavírus. Esse medicamento é bastante eficaz contra células primárias do pulmão, diminuindo também a carga viral e sinais clínicos apresentados pela doença (Madjd, 2019).

O Remdesivir teve atividade inibitória superior em comparação com a combinação de lopinavir / ritonavir e interferon $\beta$ contra MERS-CoV em modelo in vitro e em camundongo. Isso significa que coma realização de mais pesquisas, ele provavelmente poderá ser utilizado como tratamento da doença. Ele foi utilizado como tratamento em alguns países como: Vietnã, Tailândia e Estados Unidos e obteve bons resultados, sem efeitos colaterais significantes (Sathyamoorthy et al, 2020).

\subsection{Ribavirina}

A Ribavirina (Tribavirin) é um fármaco antiviral sintético análogo da guanosina, este medicamento possui amplo espectro e é utilizado contra vírus de RNA e DNA, como hepatite C e E. Ele inibe a síntese de RNA viral por meio da depleção de pools de GTP celulares na forma de monofosfato. O tratamento não funcionou e causou uma toxicidade significativa, provocando também o rompimento da membrana das hemácias (hemólise). Também foram realizados testes in vitro da mesma medicação, em pequenas concentrações combinado com o fármaco interferon (Della-Torre et al. 2020). 


\section{10 Medicamentos utilizados para Influenza}

O Favipiravir é um medicamento antiviral inibidor da RNA polimerase dependente de RNA (RdRp) em atividade contra a gripe e vários outros vírus de RNA. Ele também consegue a inibição da atividade da RNA polimerase, obtendo assim grande potencial antiviral. Atualmente está envolvido em diversos ensaios clínicos para a Covid 19(Madjd, 2019).

O Oseltamivir (Tamiflu) é um antiviral inibidor da neuraminidase que reduz a replicação da influenza e previne o comprometimento da função de barreira epitelial e da citotoxicidade. Durante a atual epidemia de SARS, o medicamento foi prescrito para sintomas de dificuldade respiratória aguda. De acordo com as pesquisas realizadas, todos os pacientes que foram tratados com o oseltamivir, inalação de oxigênio e antibióticos tiveram alta (Sathyamoorthy et al. 2020).

O Umifenovir, que é um agente antiviral oral de amplo espectro sintético, que auxilia em prevenir e curar influenza A e B, bem como hepatite B e C. Utilizado também para aumentar efetivamente a vida dos indivíduos infectados com o vírus da imunodeficiência humana (HIV) e linfócitos CD4 expectativa. Ele causa uma grande diminuição de pneumonia e reduz também a taxa de mortalidade (Madjd, 2019).

\subsection{Teicoplanina com ou sem associações}

A Teicoplanina é um antimicrobiano que atua na etapa do ciclo de vida viral inibindo a clivagem de baixo $\mathrm{pH}$, da proteína de pico viral pela catepsina L e nos endossomos tardios impedindo assim a liberação de RNA viral genômico e a continuação da replicação do vírus. Esses resultados são preliminares e precisam ser confirmados agora por um ensaio clínico randomizado (Baron, 2020).

\subsection{Hidroxicloroquina ou Cloroquina associada a Azitromicina}

A Hidroxicloroquina é um fármaco usado no tratamento e prevenção de malária sensível à cloroquina. Pode ser utilizada no tratamento de artrite reumatoide, lúpus eritematoso, cutânea tarda, febre Q, porfiria e doenças fotossensíveis, na figura 1 observamos a forma estrutural do farmaco (The American Society of Health, 2020).

Figura 1. Estrutura molecular da cloroquina e hidroxicloroquina.

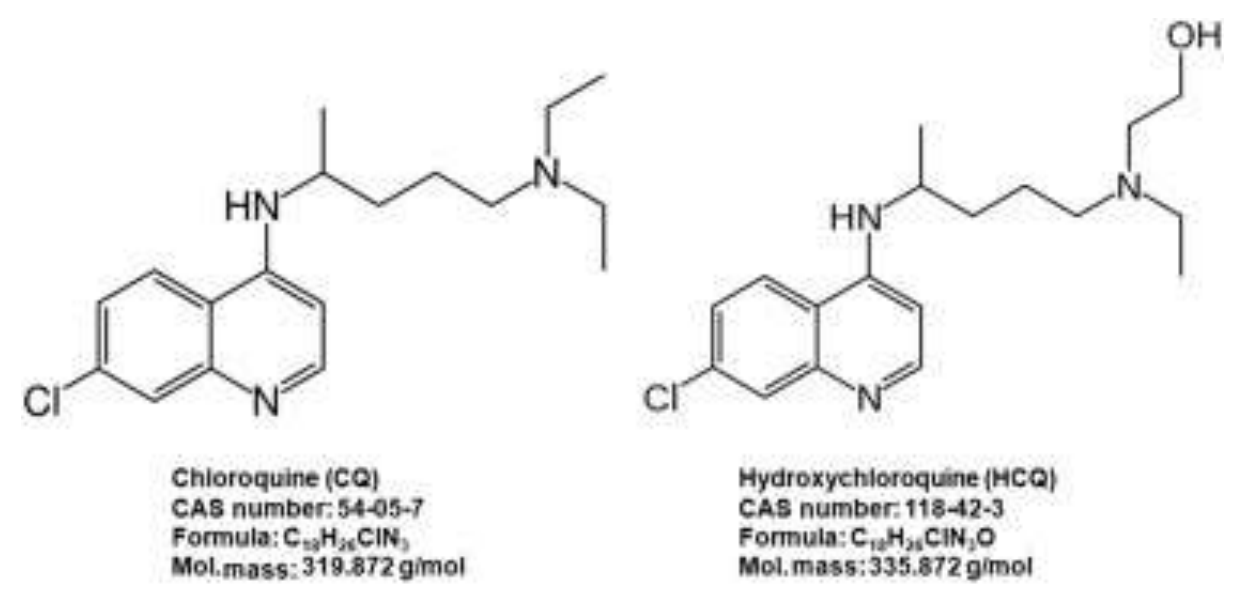

Fonte: $\mathrm{Xu}$ (2016).

Azitromicina antibiótico da classe macrolídeos, da mesma classe da Eritromicina, Claritromicina, dentre outros. Utilizados para infecções bacterianas em diversos sítios, na Figura 2 podemos observar sua composição química através da sua estrutura molecular (Anvisa, 2020). 
Figura 2. Estrutura molecular da Azitromicina.

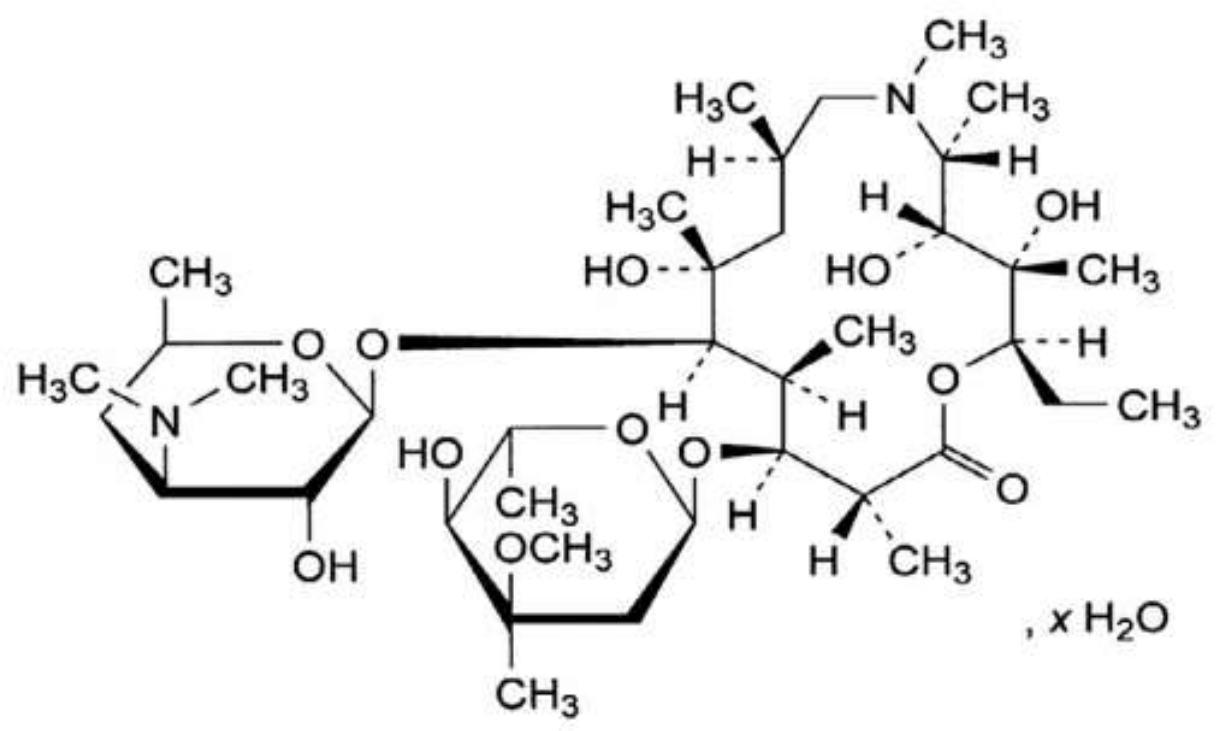

Fonte: Bakheit (2014).

Muitos estudos demonstraram o efeito da cloroquina contra o coronavirus, intermediada por receptores celulares e pelo aumento do $\mathrm{pH}$, além dessa atividade antiviral, a cloroquina, tradicionalmente um imunomodulador, emergiu como promissora no tratamento da pneumonia, que ocorre nas primeiras semanas após o início dos sintomas, porém logo após se houve controvérsias sobre o tema (Menezes et al. 2020).

Não existe muita evidencia sobre pesquisas com o fármaco, portanto há necessidade de novos ensaios clínicos. Muitas pesquisas correlacionam esses fármacos a problemas cardíacos como efeito colateral grave. Tudo isso dificulta ainda mais a sua utilização como tratamento (Atallah, 2020).

A azitromicina é um antibiótico utilizado para o tratamento de bactérias gram positivas, sendo que já demonstrou eficácia para vários vírus como o Ebola, vírus influenza, flavivírus, vírus da hepatite C, vírus do HIV e em coronavírus, como MERS-CoV e SARS-CoV (Baron, 2020).

A principal medida de prevenção quando a transmissão ainda não é comunitária é o isolamento do paciente e de todos os que entraram em contato com o mesmo, e os que tiveram contato realizar o exame e entrar imediatamente em quarentena até o recebimento do laudo (Secretaria de Atenção Primária à Saúde, 2020a).

Quando a transmissão é comunitária, ou seja, não sabemos quem transmitiu, toda a população com suspeita e com sintomas devem realizar o exame e entrar em quarentena até o recebimento do resultado (Ibidem).

A higienização de locais, utilização de mascaras nos suspeitos, utilização de EPI’S para profissionais da saúde, isolamento, evitar contato com pacientes infectados ou com sintomas, lavagem das mãos, utilização de álcool gel nas mãos e superfícies, dentre outros métodos, podem evitar a transmissão da doença (Secretaria de Atenção Primária à Saúde, 2020b).

\subsection{Plano de Contingência Nacional para Infecção Humana pelo Coronavírus COVID-19}

Existem 3 tipos de Níveis de resposta são eles: Alerta, Perigo Iminente e Emergência em Saúde Pública. O nível alerta quando existe grande risco no local para SARS-COV-2, porém não possui casos suspeitos. O Nível de resposta perigo iminente trata-se de presença de casos suspeitos de acordo com Capítulo IV, Seção I, Artigo 15 da Lei no 8.080 de 19 de setembro de 
1990, que dispõe sobre as condições para a promoção, proteção e recuperação da saúde, a organização e o funcionamento dos serviços correspondentes e precauções (Centro de Operações de Emergências em Saúde Pública, 2020).

Já o nível de resposta Emergência em Saúde Pública corresponde a situação de transmissão no local e já possui casos confirmados, esse nível é dividido em duas situações, são elas: Fase de contenção e Fase de mitigação. Na fase de contenção se há abastecimento de EPI'S, quarentena domiciliar em casos leves, evitar a ocupação de leitos desnecessariamente, aumentar a vigilância epidemiológica e atenção especializada em saúde. Na fase de mitigação ocorre a partir de 100 casos positivos para coronavirus e óbitos, medidas restritivas individuais, isolamento, quarentena, contratação emergencial de leitos de UTI (Ibidem).

\section{Considerações Finais}

A Covid 19 é uma doença nova e ainda pouco conhecida até a presente data, esta doença já levou milhares de pessoas a óbito, uma verdadeira ameaça à saúde pública. O processo de criação de novos fármacos é demorado e exige muita cautela, sendo assim os cientistas veem a necessidade de investir e realizar pesquisas com diversos fármacos na tentativa incessante de chegar a um tratamento eficaz e seguro, por isso fármacos já existentes e aprovados, que já são utilizados para outros vírus ou doenças, estão sendo experimentados para o vírus. Contudo, devemos analisar inicialmente testes in vitro logo após os in vivo para a doença. Onde devem ser considerados seus efeitos adversos, sua eficácia e a condição clínica do paciente.

Mediante aos resultados obtidos nesta pesquisa continuamos sem tratamento específico para a Covid19, foram utilizados medicamentos que pudessem amenizar os sintomas da síndrome gripal da doença. Para os casos emergenciais com piora clinica elevada ou pacientes pertencentes ao grupo de risco foram utilizados a combinação do antibiótico azitromicina e da hidroxicloroquina que é utilizado atualmente para malária. O uso desses fármacos levou a possível melhora ou até mesmo à cura de pacientes em todo mundo, porém, não conseguimos ainda garantir comprovações científicas de que a cura ou o tratamento eficaz tenha sido devido ao uso das medicações citadas neste trabalho. Isso mostra que as pesquisas devem ser ainda mais intensificadas com o intuito de conseguir comprovar cientificamente o uso eficaz e seguro destes fármacos.

A maioria desses fármacos não obtiveram eficácia, ou ainda estão em estudos prolongados, isso prova que necessitamos de mais trabalhos e pesquisas na utilização desses fármacos e outros possíveis fármacos para tratamento da doença, para chegarmos ao fármaco que tanto esperamos para o tratamento dessa doença.

\section{Referências}

Atallah, A. N., Puga, M. E. D. S., Amaral, J. L. G. S. D., \& Person, O. C. (2020). intervenção com cloroquina/hidroxicloroquina com ou sem azitromicina para covid-19 (sars-cov 2): sinopse baseada em evidências. Revista diagnóstico e tratamento. 25 (2): 01-07.

Bakheit, A. H., Al-Hadiya, B. M., \& Abd-Elgalil, A. A. (2014). Azithromycin. Profiles of drug substances, excipients, and related methodology, 39 (1), 1-40.

Baron, S. A., Devaux, C., Colson, P., Raoult, D., \& Rolain, J. M. (2020). Teicoplanin: an alternative drug for the treatment of COVID19?. International journal of antimicrobial agents, 55(4), 105944.

Centro de operações de emergências em saúde pública - COE-covid (2020). Plano de Contingência Nacional para Infecção Humana pelo novo Coronavírus COVID-19. Ministério da Saúde.

Chan, J. F., Yuan, S., Kok, K. H., To, K. K., Chu, H., Yang, J. et al. (2020). A familial cluster of pneumonia associated with the 2019 novel coronavirus indicating person-to-person transmission: a study of a family cluster. Lancet (London, England), 395 (10223), 514-523.

Della-Torre, E., Campochiaro, C., Cavalli, G., De Luca, G., Napolitano, A., La Marca, S. et al. (2020). Interleukin-6 blockade with sarilumab in severe COVID-19 pneumonia with systemic hyperinflammation: an open-label cohort study. Annals of the rheumatic diseases, 79 (10), $1277-1285$.

Elfiky A. A. (2020). Ribavirin, Remdesivir, Sofosbuvir, Galidesivir, and Tenofovir against SARS-CoV-2 RNA dependent RNA polymerase (RdRp): A molecular docking study. Life sciences, 253 (1), 117592. 
Filho, A., J. B. A., Valente, M. Y. S., Nathan, A. C., Guido, G. C., Higa, C. N. (2020). Pneumonia por COVID-19: qual o papel da imagem no diagnóstico? J. Bras. Pneumol. 46 (2).

George, P. M., Wells, A. U., \& Jenkins, R. G. (2020). Pulmonary fibrosis and COVID-19: the potential role for antifibrotic therapy. The Lancet. Respiratory medicine, 8(8), 807-815.

Hydroxychloroquine Sulfate Monograph for professional. (2020). The American Society of Health-System Pharmacists.

Imperador, C.H.L., Espreafico Junior, C.R., Nascimento, A.M.V. (2020). Cloroquina e hidroxicloroquina associado ao zinco e/ou azitromicina na COVID-19. Revista Ulakes. 1 (1), 67-73.

Lana, R. M., Coelho, F. C., Gomes, M. F. C., Cruz, O. G., Bastos, L. S., Villela, D. A. M., Codeço, C. T. 2020. Emergência do novo coronavírus (SARS-CoV-2) e o papel de uma vigilância nacional em saúde oportuna e efetiva. Cad. Saúde Pública. 36 (3), 1-5.

LTDA. (2013). Medley Farmacêutica. frmVisualizarBula.asp. Anvisa.

Madjd, Zahra. The Lancet Infectious Diseases Spotlight of Remdesivir in comparison with Ribavirin, Favipiravir, Oseltamivir and Umifenovir in Coronavirus Disease 2019 (COVID-19). The lancet. ID-D-20-01886.

Menezes, C.R., Sanches, C., Chequer, F.M.D. (2020). Efetividade e toxicidade da cloroquina e da hidroxicloroquina associada (ou não) à azitromicina para tratamento da COVID-19. O que sabemos até o momento? Journal of Health \& Biological Sciences. 8 (1), 1-9.

Ministério da saúde. (2020). Secretária de Vigilância em saúde. Centro de operações de emergência de saúde pública. Boletim Epidemiológico Especial - COE Coronavírus.

Nobre, A. F. S., Sousa, R. C. M., Santos, M. C., Barbagelata, L. S., Júnior, E. C., Lima, D. F., et al. (2014). Primeira detecção de coronavírus humano associado à infecção respiratória aguda na Região Norte do Brasil. Rev Pan-Amaz Saúde, 5 (2), 37-41.

Pereira, A. S., Shitsuka, D. M., Parreira, F. J., \& Shitsuka, R. Metodologia da pesquisa científica. UFSM.

Sandler, R. (2020). FDA aprova cloroquina e hidroxicloroquina para tratamento emergencial de coronavírus. Recuperado: https://forbes.com.br/colunas/2020/03/fda-aprova-cloroquina-e-hidroxicloroquina-para-tratamento-emergencial-de-coronavirus/>.

Sathyamoorthy, N., Chintamaneni, P. K., \& Chinni, S. (2020). Plausible role of combination of Chlorpromazine hydrochloride and Teicoplanin against COVID-19. Medical hypotheses, 144, 110011.

Secretaria de Atenção Primária à Saúde (SAPS) - Ministério da Saúde. (2020). Protocolo de manejo clínico do coronavírus (COVID-19) na atenção primária à saúde.

Secretaria de Atenção Primária à Saúde (SAPS) - Ministério da Saúde. (2020b). Manejo de corpos no contexto do novo coronavírus COVID19.

Secretaria do Estado de São Paulo. (2020a). Protocolo laboratorial para a coleta, acondicionamento e transporte de amostras biológicas para investigação de COVID-19.

Stebbing, J., Phelan, A., Griffin, I., Tucker, C., Oechsle, O., Smith, D., \& Richardson, P. (2020). COVID-19: combining antiviral and antiinflammatory treatments. The Lancet. Infectious diseases, 20 (4), 400-402.

Wu, C., Liu, Y., Yang, Y., Zhang, P., Zhong, W., Wang, Y. et al. (2020). Analysis of therapeutic targets for SARS-CoV-2 and discovery of potential drugs by computational methods. Acta pharmaceutica Sinica. B, 10 (5), 766-788.

Wu, T. C., Sacilotto, L., Darrieux, F. C. C., Pisani, C. F., Melo, S. L., Hachul, D. T. et al. (2020). Controle do Intervalo QT para Prevenção de Torsades de Pointes Durante uso de Hidroxicloroquina e/ou Azitromicina em Pacientes com COVID 19. Arquivos Brasileiros de Cardiologia. 114 (6), 1061-1066.

Xu, C., Zhu, L., Chan, T., Lu, X., Shen, W., Madigan, M. C. et al. (2016). Chloroquine and Hydroxychloroquine Are Novel Inhibitors of Human Organic Anion Transporting Polypeptide 1A2. Journal of pharmaceutical sciences, 105(2), 884-890. 